\title{
Antagonist co-activation during short and medium latency responses in subjects with chronic ankle instability
}

\author{
Andreia S.P. Sousa \\ Área Científica de Fisioterapia, Escola Superior de Saúde do Porto, Instituto Politécnico do Porto, Centro de Investigação em Reabilitação - Centro de Estudos de Movimento \\ e Atividade Humana, Rua Dr. António Bernardino de Almeida, 400, 4200-072 Porto, Portugal
}

\begin{abstract}
A B S T R A C T
Purpose: This study aims to evaluate bilateral ankle antagonist co-activation during compensatory postural responses, including short latency responses (SLR) and medium latency responses (MLR), in response to a unilateral sudden ankle inversion in subjects with chronic ankle instability (CAI).

Methods: Twenty-four participants with CAI and 20 controls participated in the present study. Bilateral eletromyography of peroneus longus (PL), peroneus brevis (PB), tibialis anterior (TA) and soleus (SOL) muscles was collected during a unilateral sudden inversion perturbation $\left(30^{\circ}\right)$ to assess antagonist co-activation of SOL/TA and TA/P pairs during SLR and MLR in both support and perturbed positions.
\end{abstract}

Results: Compared to control group, participants with CAI presented: (1) bilateral increased antagonist co-ac-tivation of SOL/TA MLR in the support position; and (2) decreased antagonist co-activation of TA/P MLR of uninjured limb in the support position and of injured limb in the perturbed position.

Conclusions: CAI involves deregulation of ankle antagonist co-activation of MLR in both injured and uninjured limbs.

Keywords: Chronic ankle instability; Postural contro; Antagonist co-activation; Short latency responses; Medium latency responses

\section{Introduction}

The analysis of reciprocal and simultaneous patterns of agonist and antagonist muscle activation is considered a fundamental way of understanding motor function (Levin and Dimov, 1997). Antagonist coactivation is defined as the simultaneous activation of both agonist and antagonist muscle groups around the joint (Levin and Dimov, 1997). Evidence suggests that its regulation is continuously controlled by the nervous system (Nielsen and Kagamihara, 1992, 1993), and that it may be centrally mediated by a descending "common drive" (De Luca and Mambrito, 1987).

It is generally accepted that a certain ratio exists between the agonist group and the antagonist group in order to maintain functional stability of the joint (Burdet et al., 2001; Hirokawa et al., 1991; Minetti, 1994). Specifically, it has been argued that an imbalance in the agonist/ antagonist ratio has a relevant influence on the severity of the posttraumatic sequelae of ankle (Lentell et al., 1990).

However, and despite the fundamental role of antagonist co-activation in the maintenance of functional joint stability, the contribution of this variable to postural balance dysfunction following acute ankle sprain has yet to be comprehensively investigated (Yeung et al., 1994). Chronic ankle instability, defined as subjective and repeated episodes of giving way and spraining of the ankle, is often the end-result of an initial ankle sprain (Hertel, 2002). It may englobe mechanical and functional deficits (Delahunt et al., 2010) and has been characterized by the presence of impaired proprioception (Docherty and Arnold, 2008; Forkin et al., 1996; Glencross and Thornton, 1981; Konradsen, 2002) and a related delayed activation timing of peroneal muscles during short latency compensatory responses (Hoch and McKeon, 2014; Konradsen and Bohsen Ravn, 1991; Lofvenberg et al., 1995; Menacho Mde et al., 2010; Mitchel et al., 2008; Munn et al., 2010b) resultant from deficits in peripheral sensory input after injury (Docherty and Arnold, 2008; Forkin et al., 1996; Glencross and Thornton, 1981; Konradsen, 2002) but also from a dysfunction in supraspinal sensorimotor control (Palmieri-Smith et al., 2009). However, despite, several studies have been developed about the postural deregulation related to CAI, neuromuscular variables have been assessed only at an individual muscle level like muscle activation timings (Hoch and McKeon, 2014; Menacho Mde et al., 2010; Munn et al., 2010a). Since postural responses involve activation of muscle synergies throughout the entire body and considering the fact that they are also more context-specific, more flexible and adaptable than spinal proprioceptive reflexes (Horak and Macpherson, 1996), it is logical to suggest that the quantification and analysis of antagonist co-activation could provide additional information about neuromuscular insufficiencies afflicting individuals with CAI. In fact, the documented persistence of alterations in motor 
control demonstrated for the injured and uninjured limbs of individuals with CAI (Doherty et al., 2014a, 2015, 2014b; Evans et al., 2004; Hass et al., 2010; Hertel and Olmsted-Kramer, 2007; McKeon and Hertel, 2008; Sousa et al., 2018; Wikstrom et al., 2009, 2010) strongly supports the hypothesis of a reorganization of central motor commands that would have repercussions in antagonist co-activation regulation. Assuming the hypothesis that the activity of all muscles within the system is interdependent (Feldman and Levin, 1995) it can be hypothesized that subjects with chronic ankle instability would present deregulation of antagonist co-activation at the ankle joint level during compensatory postural responses to an external perturbation.

Therefore, the purpose of this study is to evaluate bilateral ankle antagonist co-activation during compensatory postural responses to a unilateral sudden ankle inversion in subjects with chronic ankle instability. Since compensatory postural responses encompass a primary burst attributed to a monosynaptic excitation, short latency response (SLR), but also by a secondary burst, medium-latency response (MLR), antagonist co-activation will be evaluated for both intervals. The high prevalence of ankle sprain in athletes (Fong et al., 2007) as well the high percentage of recurrence (47-73\%) (Yeung et al., 1994) turn relevant the study of this condition in this population.

\section{Methods}

\subsection{Design}

Case control study.

\subsection{Participants}

Twenty-four athletes (6 women, 18 men) with unilateral CAI and twenty uninjured athletes ( 3 women, 17 men) participated in this study (Table 1). All volunteers were federated athletes practicing sports with high risk of ankle sprain, including soccer $(n=27)$, basketball $(n=1)$, volleyball $(n=12)$ and handball $(n=3)$. Participants assigned to the CAI group met the criteria set by the International Ankle Consortium (Gribble et al., 2014a, 2013, 2014b). For inclusion in the CAI group, subjects had to meet the following criteria: (1) history of at least one significant unilateral ankle sprain; (2) the initial sprain must have occurred at least 12 months prior to enrolment in the study; (3) at least one ankle sprain was associated with inflammatory symptoms; (4) at least one ankle sprain created at least one day of interruption of desired

Table 1

Mean and standard deviation (SD) values of age, height and weight of control and CAI groups.

\begin{tabular}{|c|c|c|c|}
\hline \multirow[t]{2}{*}{ Variables } & \multicolumn{2}{|l|}{ Mean (SD) } & \multirow[t]{2}{*}{$\mathrm{p}$-value } \\
\hline & Control & CAI & \\
\hline Age (years) & $21.8(2.21)$ & $20.6(2.52)$ & 0.098 \\
\hline Height (m) & $1.78(0.09)$ & $1.76(0.09)$ & 0.800 \\
\hline Body weight (kg) & $73.8(11.5)$ & $70.0(11.3)$ & 0.650 \\
\hline Classification of CAI & - & $\begin{array}{l}\text { FAI, } \mathrm{n}=14 \\
\text { MAI, } \mathrm{n}=10\end{array}$ & - \\
\hline $\begin{array}{l}\text { Number of previous ankle } \\
\text { sprains }\end{array}$ & - & $3.1(1.53)$ & - \\
\hline Frequency of giving way & - & $\begin{array}{l}\text { Rarely, } \mathrm{n}=8 \\
\text { Frequently, } \mathrm{n}=10 \\
\text { Often, } \mathrm{n}=6\end{array}$ & - \\
\hline Severity of ankle sprain & - & $\begin{array}{l}\text { Severe ankle sprain, } \\
\mathrm{n}=1 \\
\text { Moderate ankle sprain, } \\
\mathrm{n}=22 \\
\text { Mild ankle sprain, } \mathrm{n}=1\end{array}$ & - \\
\hline \multirow{2}{*}{$\begin{array}{l}\text { Time since last sprain } \\
\text { (months) }\end{array}$} & - & $9.0(2.90)$ & - \\
\hline & $n=20$ & $n=24$ & \\
\hline
\end{tabular}

physical activity; (5) the most recent injury must have occurred more than three months prior to enrolment in the study; and (6) history of the previously injured ankle joint "giving way" and/or recurrent sprain and/or "feelings of instability". To meet this last criterion, individuals must have answered "yes" to question 1 ("Have you ever sprained an ankle?") along with "yes" to at least four questions related to perceived ankle instability and giving-way episodes: '(2) "Does your ankle ever feel unstable while walking on a flat surface?" (3) "Does your ankle ever feel unstable while walking on uneven ground?" (4) "Does your ankle ever feel unstable during recreational or sport activity?" (5) "Does your ankle ever feel unstable while going up stairs?" (6) "Does your ankle ever feel unstable while going down stairs?". The CAI group included subjects presenting mechanical ankle instabiliy and/or functional ankle instability. Subjects were considered to have mechanical ankle instability if they presented one or more of the following conditions: (1) presence of pain or changes in talocrural joint mobility higher than $3 \mathrm{~mm}$ in the anterior drawer manual stress test (assessed using a triaxial accelerometer), compared to the uninjured side; and/or (2) talar tilt higher than $7^{\circ}$ together with a difference higher than $0^{\circ}$ in relation to the contralateral (uninjured) ankle (assessed using an electrogoniometer). The exclusion criteria for the CAI group met the criteria set by the International Ankle Consortium (Gribble et al., 2014b) and included: (1) history of previous surgeries to the musculoskeletal structures in either limb of the lower extremity; (2) history of fracture in either limb of the lower extremity requiring realignment; (3) acute injury to musculoskeletal structures of other joints of the lower extremity in the previous three months, which impacted joint integrity and function resulting in at least one day of interruption of desired physical activity; and (4) history of bilateral ankle sprain.

Healthy control participants were selected according to the same exclusion criteria applied to the CAI group and were also excluded if they had history of ankle sprain. Prior to testing, subjects were asked to identify the dominant limb, which was described as the leg which they would use to kick a ball. As no differences were observed between the dominant and the non-dominant limb of healthy subjects in a previous study that used a similar protocol to the one used in the present study (Mitchel et al., 2008), in the healthy control group this limb was selected for evaluation. In the CAI group both limbs were evaluated.

The study was approved by the local ethics committee and was implemented according to the Declaration of Helsinki. All subjects gave their written consent to participate.

\subsection{Instruments}

The activity of the agonist muscles for active ankle stability, peroneus longus (PL), peroneus brevis (PB), tibialis anterior (TA) and soleus (SOL) muscles, was assessed by electromyography. The electromyographic signal of these muscles was monitored using a bioPLUX research wireless signal acquisition system. The signals were collected at a sampling frequency of $1000 \mathrm{~Hz}$ and were preamplified in each electrode and then fed into a differential amplifier with an adjustable gain setting $(25-500 \mathrm{~Hz}$; common-mode rejection ratio: $110 \mathrm{~dB}$ at $50 \mathrm{~Hz}$, input impedance of $100 \mathrm{M} \Omega$ and gain of 1000). Self-adhesive silver chloride electromyographic electrodes were used in a bipolar configuration with a distance of $20 \mathrm{~mm}$ between detection surface centers. The skin impedance was measured with an electrode impedance checker. The electromyography and force platform signals were analysed with the Acqknowledge software (version 3.9; Biopac Systems, Inc, 42 Aero Camino Goleta, CA 93117).

The Ankle Instability Instrument was designed to classify patients with functional ankle instability and has been shown to be a reliable and valid tool (Docherty et al., 2006). The instrument presents high values of test-retest reliability (ICC $=0.95$ ). Internal consistency reliability estimates (alpha coefficients) for each factor and the total measure ranged from 0.74 to 0.83 .

A tilt platform was used to force $30^{\circ}$ of subtalar joint inversion. The 


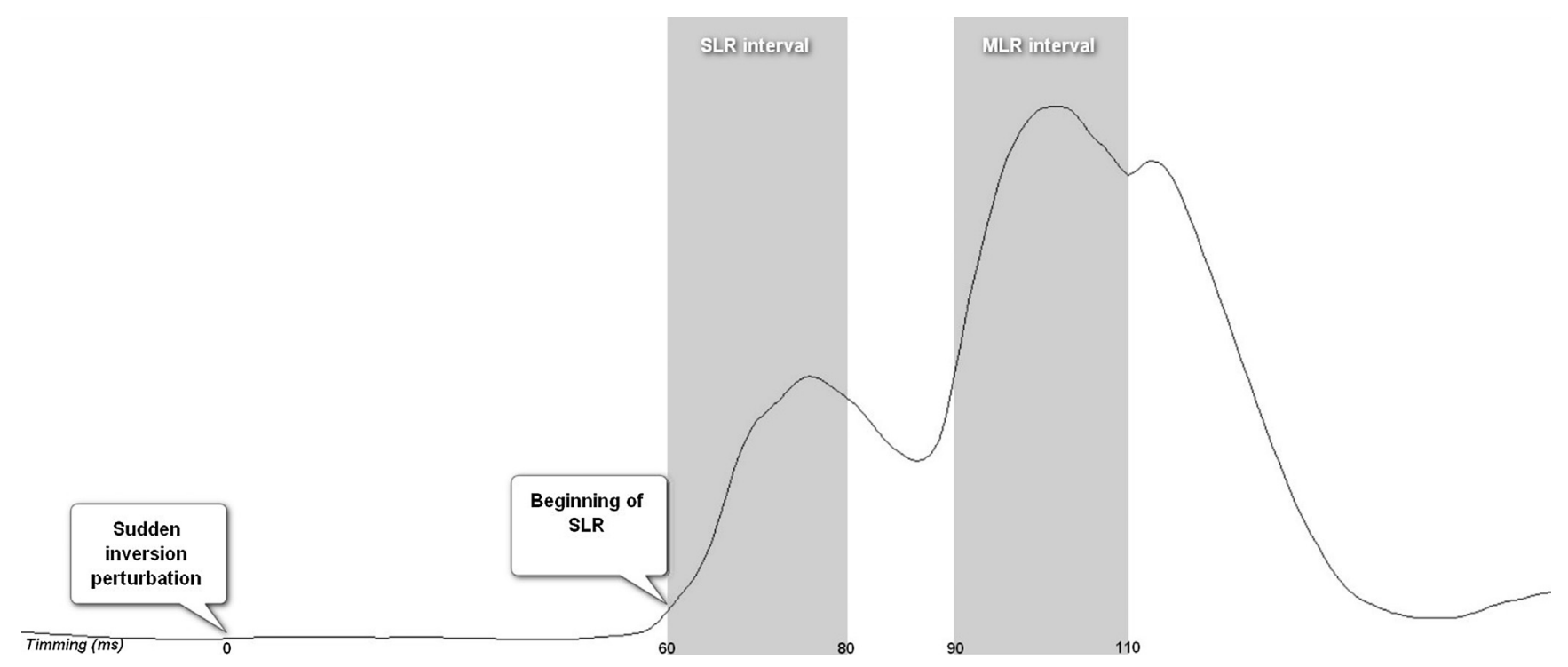

Fig. 1. Representation of a single trial of data with the identification of the timing of SLR and the interval of SLR and MLR for PL while assuming the perturbed position in a participant without CAI.

Table 2

Mean, SD and MDD values obtained for the magnitude of ankle antagonist co-activation of SLR in control and CAI groups in support and in perturbed positions. Pvalues $<0.05$ are identified in bold.

\begin{tabular}{|c|c|c|c|c|c|c|c|c|c|c|c|c|}
\hline \multirow[t]{2}{*}{ Group } & \multirow[t]{2}{*}{ Position } & \multirow[t]{2}{*}{ Muscles pair } & \multicolumn{2}{|c|}{ Uninjured limb } & \multicolumn{2}{|c|}{ Between-subjects comparisons } & \multicolumn{2}{|l|}{ Injured limb } & \multicolumn{2}{|c|}{ Between-subjects comparisons } & \multicolumn{2}{|c|}{ Within-subjects comparisons } \\
\hline & & & Mean (SD) & MDD & $\mathrm{t}$ & $\mathrm{p}$ & Mean (SD) & MDD & $\mathrm{t}$ & $\mathrm{p}$ & $\mathrm{t}$ & $\mathrm{p}$ \\
\hline Control & Support & SOL/TA & $21.6(16.35)$ & 10.4 & -1.494 & 0.145 & - & & 4.884 & 0.404 & - & \\
\hline CAI & & & 28.7 (11.59) & 7.8 & & & $25.3(11.50)$ & 6.8 & & & 0.182 & 0.858 \\
\hline Control & Perturbed & & $30.9(16.51)$ & 11.2 & 1.425 & 0.164 & - & & -0.031 & 0.975 & - & \\
\hline CAI & & & $23.1(11.96)$ & 8.8 & & & $29.4(3.60)$ & 8.4 & & & -1.652 & 0.127 \\
\hline Control & Support & $\mathrm{TA} / \mathrm{P}$ & $47.4(20.19)$ & 13.5 & 1.394 & 0.173 & - & & -1.223 & 0.229 & - & \\
\hline CAI & & & $39.6(12.29)$ & 8.0 & & & $47.1(16.62)$ & 10.2 & & & -1.129 & 0.278 \\
\hline Control & Perturbed & & 39.7 (20.89) & 13.3 & -0.207 & 0.837 & - & & -0.211 & 0.834 & - & \\
\hline CAI & & & $41.0(15.85)$ & 10.1 & & & 31.9 (13.02) & 7.5 & & & 3.242 & 0.005 \\
\hline
\end{tabular}

platform included two movable plates (trapdoors) so that either foot could be tilted independently, thus removing any subject anticipatory effect. A triaxial accelerometer (model ACC 18012018; Biosignals Research, Av. 5 de Outubro, 70 - 8 , 1050-059 Lisbon, Portugal) connected to a wireless signal acquisition system was placed in each movable plate to detect the onset of the tilt mechanism (first deflection of the accelerometer signal). For safety reasons, the tilt platform was surrounded by a handrail to the front and both sides of the subject and an adhesive, non-slip material was placed to create a footpath and to prevent slipping when the trapdoors were dropped.

\subsection{Procedures}

The skin surface of the selected muscles mid-belly and of the patella was prepared (shaved, dead skin cells and non-conductor elements were removed with alcohol and with an abrasive pad) to reduce the electrical resistance to less than $5000 \Omega$. The placement of electrodes for recording EMG activity was based on recommendations reported in the literature (Hermens et al., 2000). For the TA the electrode was placed in the $1 / 3$ on the line between the tip of the head of the fibula and the tip of the medial malleolus. For the SOL the electrode was placed $2 \mathrm{~cm}$ distal to the lower border of the medial gastrocnemius muscle belly and $2 \mathrm{~cm}$ medial to the posterior midline of the leg. For the PB the electrode was placed 1/4 on the line between the tip of the head of the fibula to the tip of the lateral malleolus. For the PL the electrode was placed anterior to the tendon of PL at $1 / 4$ of the line from the tip of the lateral malleolus to the fibula-head.
All individuals were asked to stand quietly with the support base aligned at shoulder width with one foot in each trapdoor, keeping their arms by their sides, and to focus on a target $2 \mathrm{~m}$ away and at eye level during $30 \mathrm{~s}$. The individuals were also instructed to ensure equal weight distribution between the two limbs. One limb at a time was randomly exposed to the unilateral simulated ankle sprain and was identified during analysis of each trial as the perturbed limb. The limb which was not exposed to the simulated ankle sprain in each trial was identified as the support limb. Each limb was exposed to the simulated ankle sprain three times in a random order. In each trial the trapdoor was randomly released by pushing a foot switch not visible for the subject. The subjects did not know the side nor the time of application of the perturbation. In the CAI group, the electromyographic signal was collected from both limbs (injured and uninjured limbs) and both where evaluated as support and perturbed limbs. In the control group, only the non-dominant limb was monitored as support limb and perturbed limb. Upon release, the platform fell down through an arch of $30^{\circ}$ which was predetermined by a mechanical stop leading to ankle subtalar inversion. Rest periods of $60 \mathrm{~s}$ were provided between trials, during which the subjects sat down while maintaining the foot position.

The electromyographic signals were filtered using a zero-lag, second-order Butterworth filter with an effective bandpass of $20-450 \mathrm{~Hz}$, and the root mean square was calculated. The muscle latency was detected in a time window from -450 to $-500 \mathrm{~ms}$ in relation to the first deflection of the accelerometer signal $\left(T_{0}\right)$. The latency for PL, PB, TA and SOL muscles of each limb (support and perturbed positions) was defined as the instant lasting for at least $50 \mathrm{~ms}$ when its 


\section{Support position}

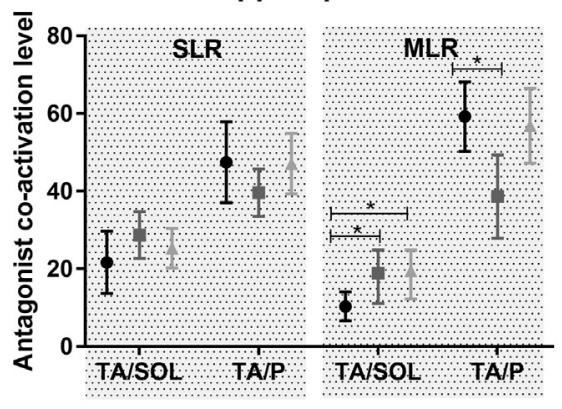

Perturbed position

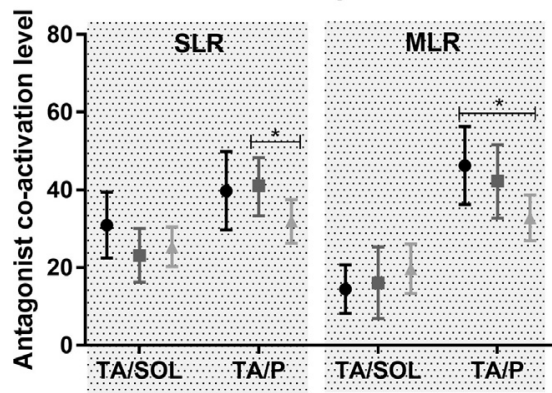

Fig. 2. Mean and 95\% confidence interval (CI) values obtained for the magnitude of ankle antagonist co-activation of SLR and MLR in control and CAI groups in support and in perturbed positions. " $\mathrm{p}<0.05$.

EMG amplitude was higher than the mean of its baseline value plus 3 standard deviations (SD), measured from -500 to $-450 \mathrm{~ms}$, using a combination of computational algorithms and visual inspection. The magnitude of overall compensatory response was evaluated over a $50 \mathrm{~ms}$ window starting at the latency of each muscle (Fig. 1). To examine the SLR and MLR, two $20 \mathrm{~ms}$ windows were defined. The first window started at the onset of the SLR (muscle latency) and the second window started $30 \mathrm{~ms}$ later. The $10 \mathrm{~ms}$ division between the two windows ensured a clear separation. The antagonist co-activation for peroneus (P) and TA muscles pair and SOL and TA muscles pair was calculated using the following equations (Kellis et al., 2003):

(a) Antagonist co-activation $\frac{T A}{P}$ pair $=\frac{E M G_{T A}}{E M G_{T A}+E M G_{P L}+E M G_{P B}} \times 100$

(b) Antagonist co-activation ${ }_{\frac{S O L}{T A}}$ pair $=\frac{E M G_{S O L}}{E M G_{S O l}+E M G_{T A}} \times 100$

\subsection{Data analysis}

The acquired data were analysed using the Statistic Package Social Science (SPSS) software from IBM Company (USA). Mean, standard deviations and $95 \%$ confidence interval (CI) were used for descriptive analysis. The minimal detectable difference (MDD) was assessed by the multiplication of the standard error of measure (SEM), 1.96 and the square root of two $(1.96 \times \sqrt{2} \times \mathrm{SEM})$.

The Independent Samples T-test was used to compare antagonist coactivation during SLR and MLR between the control and the CAI groups. The Paired Samples T-test was used to compare antagonist co-activation of SLR and MLR between injured and uninjured limbs of the CAI group. The Shapiro-Wilk test and the histogram analysis method indicated that data were normally distributed. A 0.05 significance level was used for inferential analysis.

\section{Results}

Table 2 present the antagonist co-activation levels of SOL/TA and TA/P pairs during SLR. No statistical significant differences were observed in antagonist co-activation levels between groups in both support (SOL/TA: $\mathrm{p}=0.145$ (uninjured limb), $\mathrm{p}=0.404$ (injured limb); TA/P: $\mathrm{p}=0.173$ (uninjured), $\mathrm{p}=0.229$ (injured limb)) and perturbed positions (SOL/TA: $\mathrm{p}=0.164$ (uninjured limb), $\mathrm{p}=0.965$ (injured limb); TA/P: $\mathrm{p}=0.837$ (uninjured), $\mathrm{p}=0.834$ (injured limb)) (Table 2). In the CAI group, differences were observed in the TA/P pair between injured and uninjured limbs in the perturbed position $(\mathrm{p}=0.005)$, Table 2. Decreased values of antagonist co-activation during SLR were observed in the injured limb (Fig. 2).

Regarding MLR (Table 3 and Fig. 2), increased antagonist co-activation of SOL/TA pair was observed in support position in both injured $(\mathrm{p}=0.025)$ and uninjured $(\mathrm{p}=0.038)$ limbs of CAI group compared to control group. A decreased antagonist co-activation of MLR in TA/P pair was observed in the uninjured $\operatorname{limb}(\mathrm{p}=0.004)$ and injured limb $(\mathrm{p}=0.014)$ in support and perturbed positions, respectively, compared to control group (Table 3 and Fig. 2).

\section{Discussion}

The results of the present study demonstrate that differences between groups for antagonist co-activation were only observed in MLR. MLR are mediated by group II afferents, through an oligosynaptic spinal pathway (Grey et al., 2001), group Ib afferents (Dietz, 1998) and group Ia afferents (Fellows et al., 1993). It has been argued that patients suffer partial deafferentation following ankle sprain, reducing reflexive

Table 3

Mean, SD and MDD values obtained for the magnitude of ankle antagonist co-activation of MLR in control and CAI groups in support and in perturbed positions. Pvalues $<0.05$ are identified in bold.

\begin{tabular}{|c|c|c|c|c|c|c|c|c|c|c|c|c|}
\hline \multirow[t]{2}{*}{ Group } & \multirow[t]{2}{*}{ Position } & \multirow[t]{2}{*}{$\begin{array}{l}\text { Antagonist } \\
\text { coactivation }\end{array}$} & \multicolumn{2}{|c|}{ Uninjured limb } & \multicolumn{2}{|c|}{$\begin{array}{l}\text { Between-subjects } \\
\text { comparisons }\end{array}$} & \multicolumn{2}{|l|}{ Injured limb } & \multicolumn{2}{|c|}{$\begin{array}{l}\text { Between-subjects } \\
\text { comparisons }\end{array}$} & \multicolumn{2}{|c|}{ Witin-subjects comparisons } \\
\hline & & & Mean (SD) & MDD & $\mathrm{t}$ & $\mathrm{p}$ & Mean (SD) & MDD & $\mathrm{t}$ & $\mathrm{p}$ & $\mathrm{t}$ & $\mathrm{p}$ \\
\hline Control & Support & SOL/TA & $10.3(6.90)$ & 4.8 & -2.174 & 0.038 & - & & -2.357 & 0.025 & - & \\
\hline CAI & & & $\begin{array}{l}18.9 \\
(12.23)\end{array}$ & 7.8 & & & $19.5(14.4)$ & 7.4 & & & -0.318 & 0.755 \\
\hline Control & Perturbed & & $14.4(12.2)$ & 8.1 & 0.315 & 0.755 & - & & -1.129 & 0.231 & - & \\
\hline CAI & & & $16.0(16.0)$ & 11.8 & & & $\begin{array}{l}19.6 \\
(13.16)\end{array}$ & 8.4 & & & -0.954 & 0.361 \\
\hline Control & Support & $\mathrm{TA} / \mathrm{P}$ & $59.2(17.0)$ & 11.7 & 3.138 & 0.004 & - & & 1.209 & 0.206 & - & \\
\hline CAI & & & $\begin{array}{l}38.6 \\
(20.10)\end{array}$ & 13.9 & & & $\begin{array}{l}56.9 \\
(18.79)\end{array}$ & 12.6 & & & -1.712 & 0.113 \\
\hline Control & Perturbed & & $\begin{array}{l}46.2 \\
(19.48)\end{array}$ & 13.1 & -0.629 & 0.534 & - & & 2.573 & 0.014 & - & \\
\hline CAI & & & $\begin{array}{l}42.2 \\
(17.80)\end{array}$ & 12.3 & & & $\begin{array}{l}32.8 \\
(13.65)\end{array}$ & 7.9 & & & 1.759 & 0.099 \\
\hline
\end{tabular}


activity that would be initiated by joint mechanoreceptors (Freeman, 1965]. A lack of proprioceptive information from partial deafferentation could chronically suppress gamma activation and desensitize the muscle spindle (Khin Myo et al., 1999). Changes observed in MLR could be related to a desensitization of muscle spindle type Ia and II fibers through this mechanism, but also from the recently demonstrated reduced input from Ib afferents in both injured and uninjured limbs of athletes with CAI (Sousa et al., 2017). Changes observed in MLR seem to have a relevant impact in postural dysfunction behind CAI considering the crucial role of these responses in the control of perturbations (Nardone et al., 1990). Indeed, it has been demonstrated that only MLR responses have a stabilising effect during perturbations of stance and that these responses are more influenced by the "postural set" (Nardone et al., 1990). It should be noted that changes in MLR in CAI group were only observed in a support position. This finding seems to demonstrate that postural control deregulation related to CAI is highly expressed in these conditions. It could be argued that this can be explained by the importance of muscle spindle type II fibers, compared to group Ia fibers, in the control of bipedal stance (Marchand-Pauvert et al., 2005). Moreover, the bilateral affection of MLR in CAI group observed in the present study can also be explained by decreased intput from muscle spindle group II fibers as the existence of crossed neural pathways fed by these fibers has been previously demonstrated (Nardone et al., 1996). However, the previously results obtained in (Fellows et al., 1993) by demonstrating that MLR responses in a sitting position are mediated by Ia afferent information acting on spinal pathways seem to contradict this interpretation. Future studies are required to understand the impact of the postural set on the afferents involved in MLR to confirm our hypothesis.

Specifically, the results of the present study demonstrate bilateral increased antagonist co-activation of MLR of SOL/TA pair in a support position and decreased antagonist co-activation of MLR in TA/P pair in the uninjured limb in the support position and in the injured lim in the perturbed position. The bilateral increase of antagonist co-activation in the SOL/TA pair could be interpreted as a strategy to increase stability as co-activation has been described as the most robust strategy to counteract perturbations (Damm and McIntyre, 2008; Milner, 2002) by increasing joint stiffness (Feldman and Levin, 1995) and at the same time is less challenging for the postural control system (Massion et al., 1999). However, despite being less efficient and accurate (Massion et al., 1999), the increased antagonist co-activation strategy in the support limb, by decreasing the degrees of freedom, could reduce the acceleration of the centre of pressure in the direction of the support limb to dampen the contralateral ankle sprain mechanism (Mitchel et al., 2008). However, future studies analysing centre of pressure displacement are required to confirm this hypothesis. It should be also considered the influence of pain after ankle sprain in decreasing agonist activity and increasing antagonist muscle activity (Lund et al., 1991). The decreased antagonist co-activation of TA/P pair in the uninjured limb while assuming a support position was accompanied by a decreased antagonist co-activation of the same pair in the injured limb in the perturbed position. This bilateral decrease of antagonist co-activation of TA/P compared to control group would be probably lead to decreased mediolateral functional ankle stability (Burdet et al., 2001; Hirokawa et al., 1991; Minetti, 1994). However, the results of the present study are insufficient to confirm this hypothesis, future studies involving centre of pressure related values are demanded.

\section{Limitations}

The non-evaluation of centre of pressure related variables is the major limitation of the present study. Future studies assessing these variables are needed to evaluate the impact of antagonist co-activation impairments of the CAI group and the compensatory strategies on postural stability measures. The non-evaluation of the level of disability of the CAI group limits the comparisons of the results obtained in the present study with the ones obtained in previous studies.

\section{Conclusion}

The results of the present study demonstrate that, compared to control group, subjects with CAI present bilateral changes of antagonist co-activation during MLR expressed through: (1) an increase of SOL/TA pair in a support position; and (2) a decrease of TA/P pair in the uninjured limb in a support position and in the injured limb in a perturbed position.

\section{References}

Burdet, E., Osu, R., Franklin, D.W., Milner, T.E., Kawato, M., 2001. The central nervous system stabilizes unstable dynamics by learning optimal impedance. Nature 414 (6862), 446.

Damm, L., McIntyre, J., 2008. Physiological basis of limb-impedance modulation during free and constrained movements. J. Neurophysiol. 100 (5), 2577-2588. https://doi. org/10.1152/jn. 90471.2008.

De Luca, C.J., Mambrito, B., 1987. Voluntary control of motor units in human antagonist muscles: coactivation and reciprocal activation. J. Neurophysiol. 58 (3), 525-542.

Delahunt, E., Coughlan, G.F., Caulfield, B., Nightingale, E.J., Lin, C.W., Hiller, C.E., 2010 Inclusion criteria when investigating insufficiencies in chronic ankle instability. Med. Sci. Sports Exerc. 42 (11), 2106-2121. https://doi.org/10.1249/MSS. 0b013e3181de7a8a.

Dietz, V., 1998. Evidence for a load receptor contribution to the control of posture and locomotion. Neurosci. Biobehav. Rev. 22 (4), 495-499. https://doi.org/10.1016/ S0149-7634(97)00035-3.

Docherty, C.L., Arnold, B.L., 2008. Force sense deficits in functionally unstable ankles. J. Orthop. Res. 26 (11), 1489-1493.

Docherty, C.L., Gansneder, B.M., Arnold, B.L., Hurwitz, S.R., 2006. Development and reliability of the ankle instability instrument. J. Athl. Train. 41 (2), 154-158.

Doherty, C., Bleakley, C., Hertel, J., Caulfield, B., Ryan, J., Delahunt, E., 2014a. Postural control strategies during single limb stance following acute lateral ankle sprain. Clin. Biomech. (Bristol, Avon) 29 (6), 643-649. https://doi.org/10.1016/j.clinbiomech. 2014.04.012.

Doherty, C., Bleakley, C., Hertel, J., Caulfield, B., Ryan, J., Delahunt, E., 2015. Single-leg drop landing motor control strategies following acute ankle sprain injury. Scand. J. Med. Sci. Sports 25 (4), 525-533. https://doi.org/10.1111/sms.12282.

Doherty, C., Bleakley, C., Hertel, J., Sweeney, K., Caulfield, B., Ryan, J., Delahunt, E., 2014b. Lower extremity coordination and symmetry patterns during a drop vertical jump task following acute ankle sprain. Hum. Mov. Sci. 38, 34-46. https://doi.org/ 10.1016/j.humov.2014.08.002.

Evans, T., Hertel, J., Sebastianelli, W., 2004. Bilateral deficits in postural control following lateral ankle sprain. Foot Ankle Int. 25 (11), 833-839.

Feldman, A.G., Levin, M.F., 1995. The origin and use of positional frames of reference in motor control. Behav. Brain Sci. 18 (4), 723-744.

Fellows, S.J., Domges, F., Topper, R., Thilmann, A.F., Noth, J., 1993. Changes in the short- and long-latency stretch reflex components of the triceps surae muscle during ischaemia in man. J. Physiol. 472, 737-748.

Fong, D.T., Hong, Y., Chan, L.K., Yung, P.S., Chan, K.M., 2007. A systematic review on ankle injury and ankle sprain in sports. Sports Med. 37 (1), $73-94$.

Forkin, D.M., Koczur, C., Battle, R., Newton, R.A., 1996. Evaluation of kinesthetic deficits indicative of balance control in gymnasts with unilateral chronic ankle sprains. J. Orthop. Sports Phys. Ther. 23 (4), 245-250. https://doi.org/10.2519/jospt.1996.23. 4.245 .

Freeman, M.A., 1965. Instability of the foot after injuries to the lateral ligament of the ankle. J. Bone Joint Surg. 47 (4), 669-677.

Glencross, D., Thornton, E., 1981. Position sense following joint injury. J. Sports Med. Phys. Fitness 21 (1), 23-27

Grey, M.J., Ladouceur, M., Andersen, J.B., Nielsen, J.B., Sinkjaer, T., 2001. Group II muscle afferents probably contribute to the medium latency soleus stretch reflex during walking in humans. J. Physiol. 534 (3), 925-933.

Gribble, P.A., Delahunt, E., Bleakley, C., Caulfield, B., Docherty, C., Fourchet, F., et al., 2014a. Selection criteria for patients with chronic ankle instability in controlled research: a position statement of the International Ankle Consortium. Br. J. Sports Med. 48 (13), 1014-1018. https://doi.org/10.1136/bjsports-2013-093175.

Gribble, P.A., Delahunt, E., Bleakley, C., Caulfield, B., Docherty, C.L., Fourchet, F., et al., 2013. Selection criteria for patients with chronic ankle instability in controlled research: a position statement of the International Ankle Consortium. J. Orthop. Sports Phys. Ther. 43 (8), 585-591. https://doi.org/10.2519/jospt.2013.0303.

Gribble, P.A., Delahunt, E., Bleakley, C.M., Caulfield, B., Docherty, C.L., Fong, D.T.-P., et al., 2014b. Selection criteria for patients with chronic ankle instability in controlled research: a position statement of the international ankle consortium. J. Athl. Train. 49 (1), 121-127. https://doi.org/10.4085/1062-6050-49.1.14.

Hass, C.J., Bishop, M.D., Doidge, D., Wikstrom, E.A., 2010. Chronic ankle instability alters central organization of movement. Am. J. Sports Med. 38 (4), 829-834. https://doi. org/10.1177/0363546509351562.

Hermens, H.J., Freriks, B., Disselhorst-Klug, C., Rau, G., 2000. Development of recommendations for SEMG sensors and sensor placement procedures. J. Electromyogr. Kinesiol. 10 (5), 361-374.

Hertel, J., 2002. Functional anatomy, pathomechanics, and pathophysiology of lateral 
ankle instability. J. Athl. Train. 37 (4), 364-375.

Hertel, J., Olmsted-Kramer, L.C., 2007. Deficits in time-to-boundary measures of postural control with chronic ankle instability. Gait Posture 25 (1), 33-39. https://doi.org/10 1016/j.gaitpost.2005.12.009.

Hirokawa, S., Solomonow, M., Luo, Z., Lu, Y., D'Ambrosia, R., 1991. Muscular co-contraction and control of knee stability. J. Electromyogr. Kinesiol. 1 (3), 199-208. https://doi.org/10.1016/1050-6411(91)90035-4.

Hoch, M.C., McKeon, P.O., 2014. Peroneal reaction time after ankle sprain: a systematic review and meta-analysis. Med. Sci. Sports Exerc. 46 (3), 546-556. https://doi.org/ 10.1249/MSS.0b013e3182a6a93b.

Horak, F.B., Macpherson, J.M., 1996. Postural orientation and equilibrium. In: Rowell, L., Sheperd, J. (Eds.), Handbook of Physiology. Oxford University Press, New York, pp. 255-292 Reprinted from (1996).

Kellis, E., Arabatzi, F., Papadopoulos, C., 2003. Muscle co-activation around the knee in drop jumping using the co-contraction index. J. Electromyogr. Kinesiol. 13 (3), 229-238. https://doi.org/10.1016/S1050-6411(03)00020-8.

Khin Myo, H., Ishii, T., Sakane, M., Hayashi, K., 1999. Effect of anesthesia of the sinus tarsi on peroneal reaction time in patients with functional instability of the ankle. Foot Ankle Int. 20 (9), 554-559.

Konradsen, L., 2002. Factors contributing to chronic ankle instability: kinesthesia and joint position sense. J. Athl Train. 37 (4), 381-385.

Konradsen, L., Bohsen Ravn, J., 1991. Prolonged peroneal reaction time in ankle instability. Int. J. Sports Med. 12 (03), 290-292. https://doi.org/10.1055/s-20071024683.

Lentell, G., Katzman, L.L., Walters, M.R., 1990. The relationship between muscle function and ankle stability. J. Orthop. Sports Phys. Ther. 11 (12), 605-611.

Levin, M.F., Dimov, M., 1997. Spatial zones for muscle coactivation and the control of postural stability. Brain Res. 757 (1), 43-59. https://doi.org/10.1016/S00068993(97)00204-7.

Lofvenberg, R., Karrholm, J., Sundelin, G., Ahlgren, O., 1995. Prolonged reaction time in patients with chronic lateral instability of the ankle. Am. J. Sports Med. 23 (4), 414-417.

Lund, J.P., Donga, R., Widmer, C.G., Stohler, C.S., 1991. The pain-adaptation model: a discussion of the relationship between chronic musculoskeletal pain and motor activity. Can. J. Physiol. Pharmacol. 69 (5), 683-694.

Marchand-Pauvert, V., Nicolas, G., Marque, P., Iglesias, C., Pierrot-Deseilligny, E., 2005. Increase in group II excitation from ankle muscles to thigh motoneurones during human standing. J. Physiol. 566 (Pt 1), 257-271. https://doi.org/10.1113/jphysiol. 2005.087817.

Massion, J., Ioffe, M., Schmitz, C., Viallet, F., Gantcheva, R., 1999. Acquisition of anticipatory postural adjustments in a bimanual load-lifting task: normal and pathological aspects. Exp. Brain Res. 128 (1-2), 229-235.

McKeon, P.O., Hertel, J., 2008. Systematic review of postural control and lateral ankle instability, Part I: can deficits be detected with instrumented testing. J. Athl. Train. 43 (3), 293-304.

Menacho Mde, O., Pereira, H.M., Oliveira, B.I., Chagas, L.M., Toyohara, M.T., Cardoso, J.R., 2010. The peroneus reaction time during sudden inversion test: systematic review. J. Electromyogr. Kinesiol. 20 (4), 559-565. https://doi.org/10.1016/j. jelekin.2009.11.007.

Milner, T.E., 2002. Adaptation to destabilizing dynamics by means of muscle cocontraction. Exp. Brain Res. 143 (4), 406-416. https://doi.org/10.1007/s00221-0021001-4.

Minetti, A.E., 1994. Contraction dynamics in antagonist muscles. J. Theor. Biol. 169 (3), 295-304. https://doi.org/10.1006/jtbi.1994.1150.

Mitchel, A., Dyson, R., Hale, T., Abraham, C., 2008. Biomechanics of ankle instability. Part 1: reaction time to simulated ankle sprain. Med. Sci. Sports. Exerc. 40 (8), 1515-1521. https://doi.org/10.1249/MSS.0b013e31817356b6.

Munn, J., Sullivan, S.J., Schneiders, A.G., 2010. Evidence of sensorimotor deficits in functional ankle instability: a systematic review with meta-analysis. J. Sci. Med. Sport 13 (1), 2-12. https://doi.org/10.1016/j.jsams.2009.03.004.

Nardone, A., Giordano, A., Corrà, T., Schieppati, M., 1990. Responses of leg muscles in humans displaced while standing. effects of effects of types of perturbation and of postural set. Brain 113 (1), 65-84. https://doi.org/10.1093/brain/113.1.65.

Nardone, A., Grasso, M., Giordano, A., Schieppati, M., 1996. Different effect of height on latency of leg and foot short- and medium-latency EMG responses to perturbation of stance in humans. Neurosci. Lett. 206 (2-3), 89-92. https://doi.org/10.1016/S03043940(96)12430-7.

Nielsen, J., Kagamihara, Y., 1992. The regulation of disynaptic reciprocal Ia inhibition during co-contraction of antagonistic muscles in man. J. Physiol. 456, 373-391.

Nielsen, J., Kagamihara, Y., 1993. The regulation of presynaptic inhibition during cocontraction of antagonistic muscles in man. J. Physiol. 464, 575-593.

Palmieri-Smith, R.M., Hopkins, J.T., Brown, T.N., 2009. Peroneal activation deficits in persons with functional ankle instability. Am. J. Sports Med. 37 (5), 982-988. https://doi.org/10.1177/0363546508330147.

Sousa, A.S.P., Costa, B., Leite, J., Santos, R., 2017. Bilateral proprioceptive evaluation in individuals with unilateral chronic ankle instability. J. Athl. Train. 52 (4), 360-367.

Sousa, A.S.P., Silva, M., Gonzalez, S., Santos, R., 2018. Bilateral compensatory postural adjustments to a unilateral perturbation in subjects with chronic ankle instability. Clin. Biomech. (Bristol, Avon) 57, 99-106. https://doi.org/10.1016/j.clinbiomech. 2018.06.015.

Wikstrom, E.A., Naik, S., Lodha, N., Cauraugh, J.H., 2009. Balance capabilities after lateral ankle trauma and intervention: a meta-analysis. Med. Sci. Sports Exerc. 41 (6), 1287-1295. https://doi.org/10.1249/MSS.0b013e318196cbc6.

Wikstrom, E.A., Naik, S., Lodha, N., Cauraugh, J.H., 2010. Bilateral balance impairments after lateral ankle trauma: a systematic review and meta-analysis. Gait Posture 31 (4), 407-414. https://doi.org/10.1016/j.gaitpost.2010.02.004.

Yeung, M.S., Chan, K.M., So, C.H., Yuan, W.Y., 1994. An epidemiological survey on ankle sprain. Br. J. Sports Med. 28 (2), 112-116. 